\title{
Research of High Voltage Circuit Breaker Test Method Based on Multiple Data Fusion
}

\author{
Shi Liu ${ }^{1}$, Jin Tan ${ }^{2}$, Mengyuan $\mathrm{He}^{2, \mathrm{a}}$ and Sun Cai ${ }^{1}$ \\ ${ }^{1}$ Guangdong Electric Power Research Institute, Guangzhou, Guangdong 510600, China \\ ${ }^{2}$ North China Electric Power University, Baoding, Hebei 071003, China \\ apriangelina@163.com
}

\begin{abstract}
Keywords: Circuit breaker, Mechanical characteristic, Multi-signal fusion, Fault diagnosis.
Abstract. This paper proposes a new type of circuit breaker mechanical characteristic test method, which breaks through the former "contact" sensing technology. It adopts the technique of multi-data fusion, combines with electrical, sound and vibration, and studies the circuit breaker mechanical characteristics represented by various characteristics in this kind of "trinity" method. This test method can provide more effective way for fault diagnosis of circuit breaker institutions, and promote the development online fault detection work of circuit breaker.
\end{abstract}

\section{Introduction}

Circuit breaker condition monitoring and fault diagnosis has always been an important topic in the field of electric at home and abroad ${ }^{[1][2][3]}$. By testing and monitoring the specific parameter of circuit breaker, the property, extent, category, and component of the equipment fault can be determined. The relationship among fault, signs, reason and system can be cleared, and the development trend of the fault can be indicated. But for typical faults of the circuit breaker such as refusing action and misoperation, the main reason is mostly in mechanical. Thus, it may be difficult to find only by general power monitoring and judgment. Before the fault is triggered, the change of mechanical properties of operation mechanism is a gradual process. If the mechanical properties can be monitored in operation, it is possible to send out warning signals before the fault, process the fault in advance, and avoid the happening of the fault ${ }^{[4][5][6]}$. Therefore, fault is analyzed by method of multiple parameters, which combines multiple signals such as the electrical, sound and vibration, expands the means of condition monitoring of operating mechanism of circuit breaker. Then the signals prove each other and the "chain of evidence" can be formed, which greatly improves the reliability of the diagnosis results. In addition, because of the adoption of "contactless" measurement technology of sound and vibration signal, online fault detection of circuit breaker can be effectively promoted.

\section{System Platform Design}

This system should provide an auxiliary method of research about the mechanical properties in the process of circuit breaker operation, so that the running state can be identified accurately. Therefore, sensor used for sound and vibration signal which is produced in the process of high voltage circuit breaker operation need to meet the requirements of a certain precision index. Data acquisition card for acoustic and vibration signal requires certain sampling rate and synchronization acquisition in order to unified time scale and analysis the signal. Computer in this system should support synchronous acquisition card and have enough memory and processing speed to analyze and process the sound and vibration signals collected. This system exhibit a certain degree of anti-interference capabilities. Meanwhile, it can satisfy the demands of devices interface standards ${ }^{[7][8]}$.

This system mainly includes hardware test platform part and software part. The hardware part mainly includes sensors, acquisition card and PC. The main function of the software part which is based on LabVIEW development platform is to read, playback, identify and handle the collected signal. 
Hardware Part. This system uses 8 slot chassis of PXIe - 1062Q type which is produced by NI Company from the United States. It has all the features of the latest PXI specification.

The selected controller of acquisition system is PXIe-8115 high performance embedded controller. Combine this controller with NI LabVIEW software system, the development efficiency can be promoted in all kinds of test, measurement and control application. The selected acquisition card is a high precision data acquisition (DAQ) module PXIe-4492 which is specially designed for sound and vibration application. PXIe-4492 has a flexible channel configuration which can provide 8 road synchronous channels in a single PXI Express module, and it can collect a variety of signal such as current, voice, vibration and so on.

Acquisition of sound signals choose high fidelity bullet pinhole type noise reduction micro sound listener. It sets up an AGC automatic gain control circuit internally to enhanced sound waves over a long distance. Its maximum scope of influence can up to 100 square meters, 20-20 KHZ frequency, and sensitivity can reach -46DdB.

Acquisition of vibration signal uses three-axis piezoelectric acceleration sensor. It has characteristics of wide frequency response, strong sensitivity, small transverse sensitivity and strong ability of resisting interference.

Current signal is measured by Hall current sensor of 0.2 -magnitude, which has high sensitivity with an opening type.

There are five road signals collected in this system, including two electrical signals, two sound signals and one vibration signal. The Hall current sensor is shown in Fig. 1. The acoustic sensor is shown in Fig. 2. The vibration sensor (3D signal) is shown in Fig. 3. The system connection box front panel is shown in Fig. 4. The overall structure of the system is shown in Fig. 5.

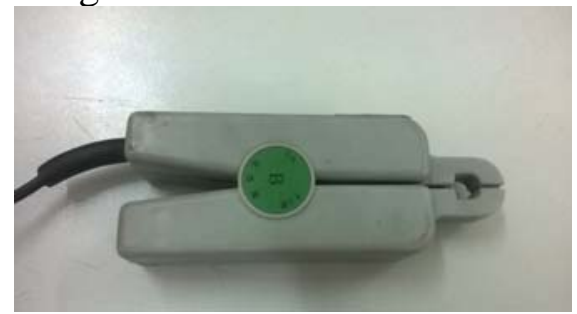

Fig. 1 The Hall current sensor

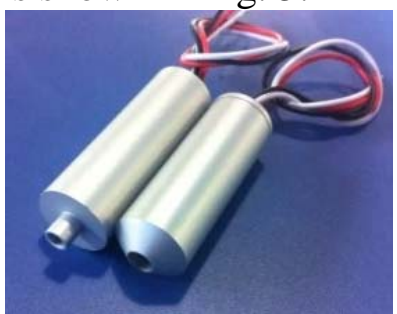

Fig. 2 The acoustic sensor

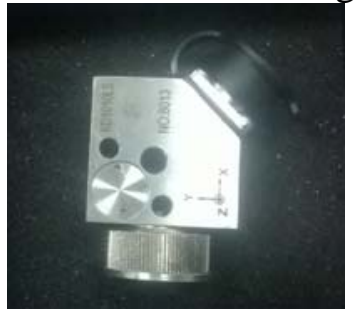

Fig. 3 The vibration sensor (3D signal)

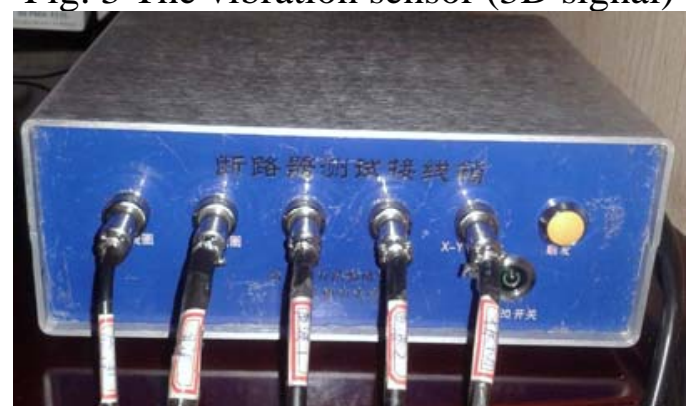

Fig. 4 The system connection box front panel 


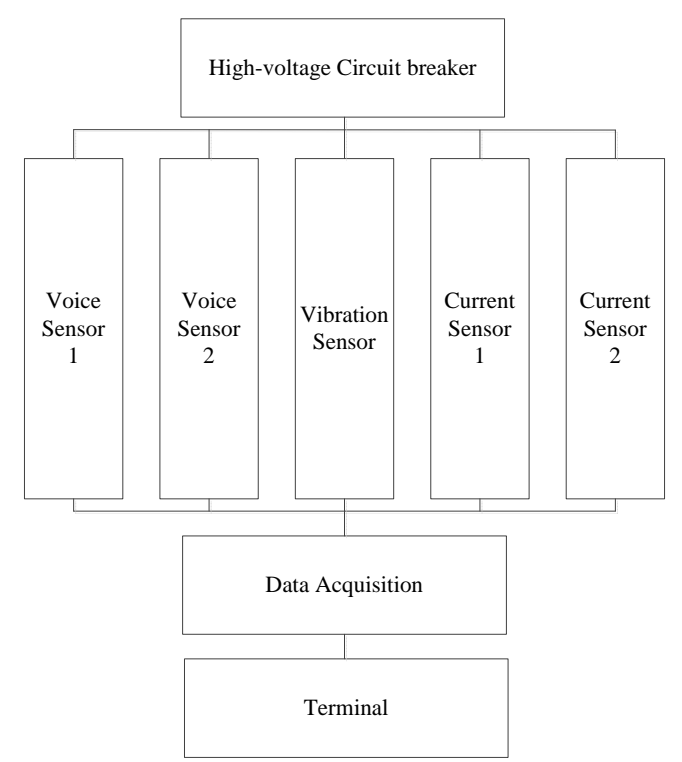

Fig. 5 Overall structure of the system

Software Part.Due to the hardware test system is PXI system which is built based on virtual instrument, so that the software adopts LabVIEW which can easily control signal collection. It not only has a great man-machine interface, but also has very powerful data processing ability.

Main functions of the software part include data acquisition, waveform display and data storage. In order to ensure that the data will not be lost, data acquisition, display and data storage will not go on at the same time. After completing the data acquisition and display, the user can choose whether to save the collected data or not.

\section{Signal Acquisition}

In the process of the entire circuit breaker action, electrical, sound and vibration signal samples 200000 points, respectively.

Electrical Signals. The two roads of the electrical signals are close and open coil current signal. Circuit curves simulated and obtained from closing data is showed in Fig. 6. Circuit curves simulated and obtained from opening data is showed in Fig. 7.

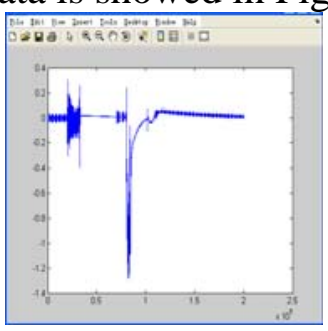

Fig. 6 Closing current curve

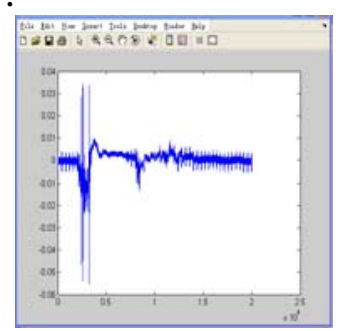

Fig. 7 Opening current curve

Sound Signals. There are two roads sound signals in all. The fitting curves are shown in Fig. 8 and Fig. 9, respectively.
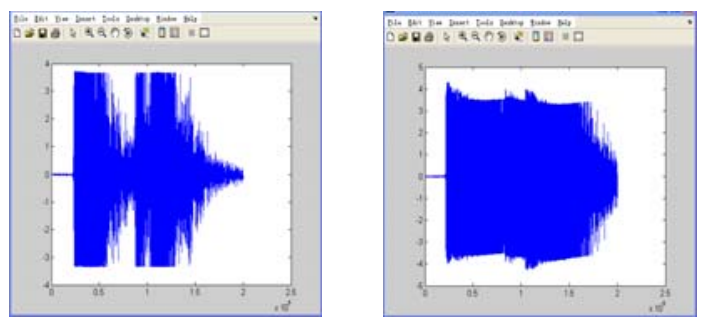

Fig. 8 The first sound signal curve Fig. 9 The second sound signal curve

Vibration Signals.Signals from $\mathrm{x}, \mathrm{y}, \mathrm{z}$ three directions were collected respectively by vibration sensor. The fitting curves can be shown respectively in Fig. 10, Fig. 11, and figure 12. 

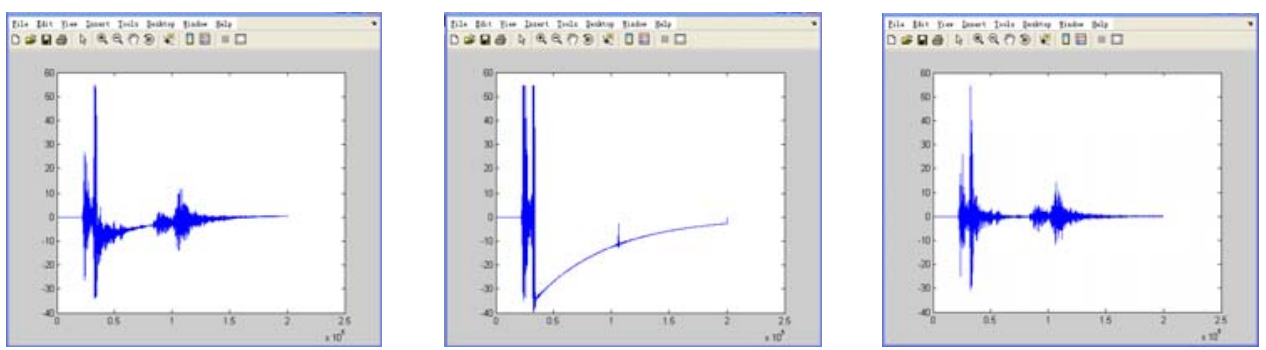

Fig. $10 \mathrm{X}$ direction signal curve Fig. $11 \mathrm{Y}$ direction signal curve Fig. $12 \mathrm{Z}$ direction signal curve

\section{Conclusions}

When circuit breaker is in the normal operation, electricity, sound and vibration signals should respectively correspond to the curve which is shown in this article. If any signal cannot correspond to the curve mentioned before, then, circuit breaker mechanical fault may occur. This paper proposes a multi-signal fusion technique. Evidences from various sources indicates a high-efficiency and accurate mechanical characteristic test method of circuit breaker, which the accuracy of faults recognition can be greatly improved.

\section{Acknowledgements}

This paper is subsidized by Southern Power Grid science-technology program (GDKJ00000031) (Research of mechanical fault diagnosis technology of circuit breaker spring operating mechanism).

\section{References}

[1]Huaijun Zhao, Peng Ma, Weiping Fu, Yuangui Cheng. Detection Device for Mechanical Characteristics of Circuit Breaker Based on DSP[J]. High Voltage Apparatus, 2006, 05:382-384.(In Chinese)

[2]Qiya Ye, Wenjin Dai, Xiuqing Han, Qiaohong Wu. Dectection and Measuremant of High Voltage Circuit Breaker for Mechanical Characteristics[J]. Jiangxi Electric Power, 2001, 01:9-11. (In Chinese)

[3]Junxia Lv, Xinchun Zhang. Operating conditions and maintenance technology of circuit breaker[J]. Precise Manafacturing \& Automation, 2011,01:62-64. (In Chinese)

[4]Chenglong $\mathrm{Xu}$, Hong Yu, Liangtai Dong, Zekun Liu, Biqiang Du. Fault identification for operating mechanism of high voltage circuit breaker in new type[J]. Yunnan Electric Power, 2014, 05:69-71. (In Chinese)

[5]Li Shen, Yulong Huang. Research of high voltage circuit breaker mechanical condition monitoring[J]. Proceedings of the CSEE, 1997, 17(2):113-117. (In Chinese)

[6]Yi Qian. Measurement for Stroke and closing speed of Vacuum circuit breaker[J]. Shanghai Electric Power, 2008, 04:390-391. (In Chinese)

[7] Su Q, Mi C, Lai L, et al. A fuzzy dissolved gas analysis method for the diagnosis of multiple faults in a circuit breaker. IEEE Transactions on Power System, 2003, 15(2):593-598.

[8] Islam S M, Wu T, Ledwich G. A novel fuzzy logic approach to circuit breakers fault diagnosis. IEEE Transactions on Dielectrics and Electrical Insulation, 2005, 7(2):177-186. 